\title{
Non-genomic progesterone receptors in the mammalian ovary: some unresolved issues
}

\author{
Tony Bramley \\ The University of Edinburgh, Division of Reproductive and Developmental Sciences, Simpson Centre for \\ Reproductive Health, Academic Centre, New Royal Infirmary, 49 Little France Crescent, \\ Old Dalkeith Road, Edinburgh EH16 4SB, Scotland, UK
}

\begin{abstract}
In addition to their well-documented genomic effects, steroid hormones may also exert actions that are: (i) rapid, (ii) insensitive to inhibitors of transcription, (iii) mimicked by steroids coupled to cell membrane-impermeant molecules, and (iv) demonstrable in cells that do not express the classic genomic progesterone receptor (gPR). Such 'non-genomic' effects have been described for all the major classes of steroids (progesterone, oestrogens, androgens and corticoids), as well as for thyroid hormones, retinoids and vitamin $\mathrm{D}_{3}$. Rapid, membrane-mediated effects of progesterone have been studied most intensively in human spermatozoa and in the Xenopus oocyte. However, similar non-genomic actions of progesterone and other steroids have now been described in a wide variety of different tissues in many species. The first putative membrane steroid receptor to be cloned was that for the pig membrane progesterone receptor (mPR). Subsequently, similar genes were cloned from rats and cattle, and two related mPRs have been described in humans. Despite accumulating evidence for cell-surface membrane actions of steroids, a number of uncertainties remain as to the properties and identity of such 'receptors' and their cellular actions. Furthermore, some rapid steroid effects may be mediated through membraneassociated 'classical' steroid receptors, and steroid receptors may be capable of activating other signalling pathways non-classically. This review focuses on some of these unresolved issues, taking as its model the actions of progesterone in the mammalian ovary.
\end{abstract}

Some of the earliest studies into steroid hormone action clearly showed that steroids could bind to specific, high-affinity binding sites (or receptors) localized on surface-membrane fractions of their target cells (Pietras and Szego, 1977). However, other laboratories demonstrated the presence of specific steroid receptors in the nuclear-cytosolic fractions of target cells and showed that such receptors could act as ligand-dependent transcription factors capable of modulating the activity of specific genes that were known to be regulated by steroid hormones. These findings led, understandably, to the concentration of effort on elaborating the classical genomic steroid hormone actions. However, there has been a recent resurgence of interest in the actions of steroids at the cell surface membrane, and a growing realization that some effects of steroid hormones clearly cannot be explained by a change in transcription (Table 1). A number of excellent reviews have appeared in the last few years that discuss in detail non-genomic effects of progesterone (Sutter-Dub, 2002) and other

Email: tbramley@staffmail.ed.ac.uk steroids (Wehling, 1997; Revelli et al., 1998; Falkenstein et al., 2000a; Schmidt et al., 2000; Kelly and Levine, 2001).

Progesterone is an important intermediate in the synthesis of androgens and oestrogens. It plays an important role in ovulation, atresia and luteinization in vivo (Telleria and Deis, 1994; Chaffin and Stouffer, 2000), and is essential for the continuation of early pregnancy in all mammalian species. However, the importance of genomic and non-genomic responses to progesterone in the mammalian ovary is still being debated. Some of the issues addressed in this review are:

- Which responses to progesterone are mediated through classical genomic effects, non-classical genomic or non-genomic actions?

- Which regions of the receptor protein or gene are detected by different antibodies or primers?

- What isoforms or variants of the genomic or membrane receptor(s) are expressed in progesterone target cells and in what proportions?

- How do cross-talk with other hormone and growth factor signalling processes, post-translational receptor 
Table 1. Comparison of some characteristics of genomic and non-genomic effects of steroid hormones

\begin{tabular}{lll}
\hline & \multicolumn{1}{c}{ Genomic effect } & \multicolumn{1}{c}{ Non-genomic effect } \\
\hline Latency of action? & Slow (normally several hours) & Rapid (seconds to minutes) \\
Inhibitors of transcription? & Effect blocked & Effect persists \\
Cells lacking a nucleus? & No effect demonstrable & Effect persists \\
Cells lack genomic receptor? & No effect demonstrable & Effect persists \\
Isolated cell membranes? & No effect demonstrable & Effects demonstrable \\
$\begin{array}{l}\text { Cell-impermeable steroid? } \\
\text { Inhibitors of 'classic' genomic } \\
\text { steroid receptor? }\end{array}$ & No effect demonstrable & Effect persists \\
\hline
\end{tabular}

modification, and type of cell influence the cellular response to progesterone?

\section{Classical genomic progesterone receptors}

The genomic progesterone receptor is a member of the ligand-inducible DNA-binding superfamily of nuclear transcription factors that includes the receptors for the other steroid hormones, thyroid hormones, vitamin D, retinoic acid and a variety of 'orphan' receptors. The cDNA for the B-form of the (human) genomic progesterone receptor (gPR-B) consists of eight different exons separated by seven short introns, and codes for a 933 amino acid (116 kDa) protein containing a number of different functional domains (Fig. 1). Exon 1 codes for the $\mathrm{N}$-terminal region of the receptor and for one of three activation function domains (AF-3). Exons 2 and 3 code for AF-1 and the DNA-binding domain (DBD) with its two zinc fingers. Exons 4-8 code for the ligand-binding domain (LBD) and AF-2. This region also codes for the hinge region, a nuclear localization signal (NLS), and regions important for receptor dimerization and binding of heat-shock proteins and immunophilins. Two other isoforms of the human $\mathrm{gPR}$ arise from the use of alternate promoters within the same gene. PR-A is N-terminally truncated (94 kDa), lacking 164 amino acids containing the AF-3 domain (Giangrande and McDonnell, 1999), whereas PR-C (60 kDa) lacks exon 1 and most of exon 2 (Wei and Miner, 1994).

Several other variants of gPR have been described (Fig. 1). One variant codes for a protein that lacks the NLS, hinge region and part of the LBD; another variant has a frameshift that gives rise to a truncated protein lacking much of the LBD (Hodges et al., 1999). In addition, other PR variants have been described that have deletions of one or more exons (Misao et al., 1998a; 2000) or that have previously unidentified $5^{\prime}$ sequences spliced to novel exons, yet still retain those exons (exons 4-8) that code for the LBD (Hirata et al., 2000, 2002). Variants lacking the LBD or DBD can modify the effects of fulllength wild-type gPR-B (Vegeto et al., 1993; Wei et al., 1997; Hodges et al., 1999; Misao et al., 2000).

\section{Effects of progesterone and anti-gestagens on ovarian function: classical genomic, non-classical genomic or non-genomic effects?}

Some of the known responses of ovarian tissues to progesterone in vitro are listed for domestic animals (Table 2 ), humans and primates (Table 3) and rats (Table 4). Both the dose-response characteristics and sensitivity to anti-progestins of rapid and longer-term responses are distinct. Stimulation of intracellular $\mathrm{Ca}^{2+}$ and inositol trisphosphate $\left(\mathrm{IP}_{3}\right)$ (and protein kinase $\mathrm{C} \beta 1(\mathrm{PKC}-\beta 1)$ ) by progesterone is detectable within seconds at picomolar levels and is not blocked by anti-gestagens. In general, longer-term actions (effects on steroidogenesis, apoptosis and proliferation; activation of protein or mRNA synthesis) are detectable only at much higher (nanomolar to micromolar) concentrations of progesterone, and are reversed by anti-gestagens (Tables 2-4), implying action via classical genomic progesterone receptors. To date, rapid $\mathrm{Ca}^{2+}, \mathrm{IP}_{3}$, phospholipase $\mathrm{C}-\beta$ (PLC- $\beta$ ) responses have been studied only in pig granulosa cells, although similar rapid and specific responses to androgen and oestrogen have also been observed in pig granulosa cells (Lieberherr et al., 1999) and to other steroids in cells lacking classical steroid receptors (Le Mellay et al., 1999). Whether rapid response to very low progesterone concentrations is a general response in the ovarian cells of other mammalian species is still unclear.

The actions of progesterone and synthetic progestagens may differ (for review, see Swan et al., 2002). Indeed, although $\left[{ }^{3} \mathrm{H}\right]$ progesterone tracer bound readily to plasma-membrane binding sites in the bovine ovary (Rae et al., 1998), 3ß-glucuronide-11- ${ }^{125}$ I-labelled tyramine-progesterone and progesterone coupled to radiolabelled BSA were poor ligands (Bramley et al., 2002). Moreover, a number of synthetic progestagen agonists (levonorgestrel, medroxyprogesterone acetate, ORG2058, R5020) and antagonists (mifepristone, onapristone) with high activity for classical gPRs failed to compete for ovarian $\left[{ }^{3} \mathrm{H}\right]$ progesterone binding sites (Rae et al., 1998), indicating that progestagenic agonists, derivatives and antagonist analogues may not behave in the same manner as progesterone itself. 


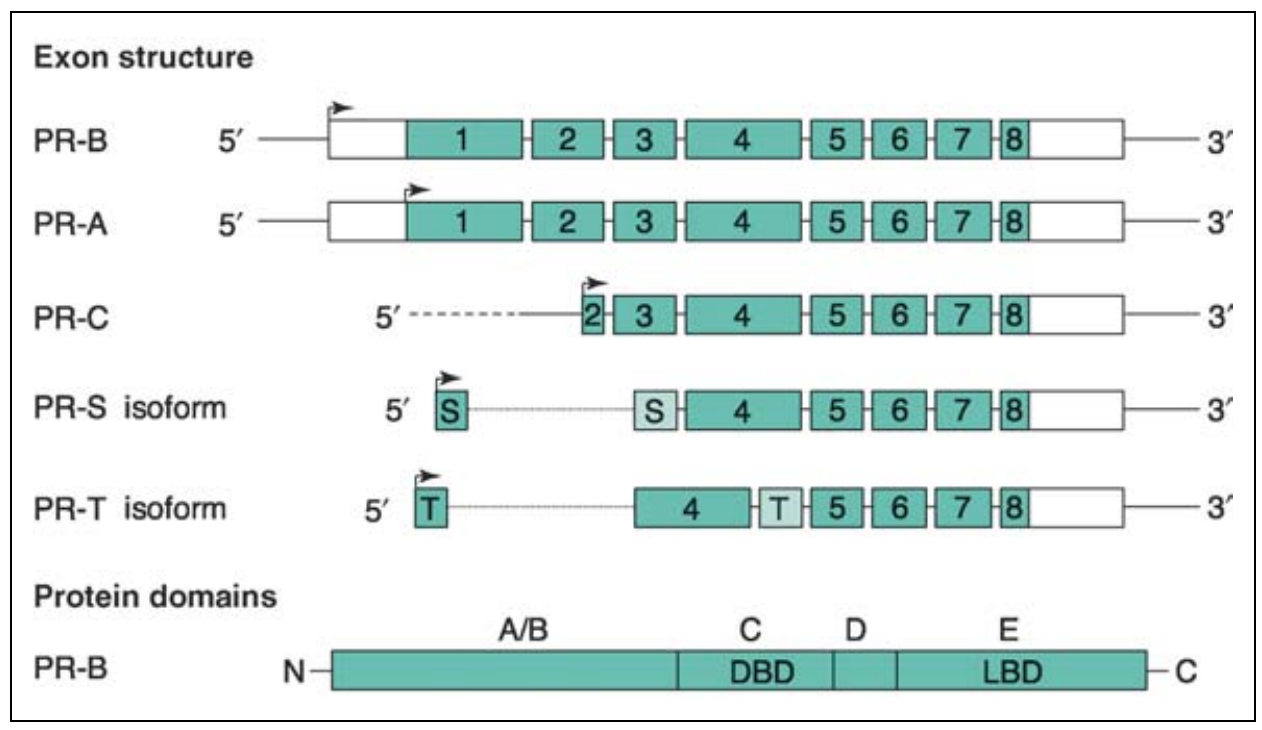

Fig. 1. Genomic structure and functional domains of the human progesterone receptor and its variants. DBD: DNA-binding domain; LBD: ligand-binding domain; PR: progesterone receptor. Arrows indicate transcription initiation sites.

\section{Ovarian genomic progesterone receptors}

The presence and cellular localization of gPR proteins and mRNA in ovarian tissues has been demonstrated by immunocytochemistry and in situ hybridization (Tables 5 and 6). Most of these studies have used antibodies and primers designed to detect either the ligand binding domain (LBD) of the gPR, or the N-terminus (to differentiate PR-A and PR-B isoforms). PR-A and PR-B have been localized simultaneously in the same types of cell (Mote et al., 1999). It will be of interest to examine the distribution of PR-A and PR-B isoforms in types of ovarian cell at different stages of the ovarian cycle, and to study their responses to luteotrophic and luteolytic stimuli.

The co-expression of PR-A or PR-C in the same type of cell as PR-B modulates its activity (Vegeto et al., 1993; Wei et al., 1997). Moreover, genomic actions of progesterone receptor isoforms are affected differentially by their association with chaperones (Smith, 2000) and nuclear coactivators (Rowan and O'Malley, 2000), and are modulated by receptor phosphorylation (Orti et al., 1992) and by cross-talk with other signalling pathways (mitogen-activated protein kinase (MAPK), Src, tyrosine kinases, phosphatidyl inositol 3-kinase (PI3-K)) that are activated by growth factors and cytokines (Richer et al., 1998; Boonyaratanakornkit et al., 2001; Bagowski et al., 2001). Furthermore, gPRs can exist in different functional states (Smith et al., 2000), and can exhibit direct nongenomic actions in their unliganded (Weigel and Zhang, 1998) and undimerized states (Cohen-Solal et al., 1993). Finally, PR-A and PR-B isoforms can activate different constellations of genes even within the same cell (Richer et al., 2002), highlighting the enormous complexity of the intracellular processes involved in progesterone activation of a target cell.

\section{Non-genomic membrane receptors for progesterone}

Steroids can affect membrane-mediated events nongenomically in a number of ways (for example, by effects on membrane fluidity, or by direct effects on other G-protein-coupled receptors; for review, see Falkenstein et al., 2000b). However, studies of rapid, steroid-specific responses to progesterone, mediated by the cell membrane, culminated in the isolation and cloning of a non-genomic (pig) progesterone receptor of 194 amino acids, with a single membrane-spanning domain (Falkenstein et al., 1996; Meyer et al., 1996). Homologous proteins were subsequently cloned in rats (Selmin et al., 1996; Krebs et al., 2000; Nolte et al., 2000) and cattle (Cenedella et al., 1999; Zhu et al., 2001), and two putative mPR homologues (hpr6.6 and Dg6) were identified in humans (Gerdes et al., 1998). The human mPR gene consists of three exons separated by two introns. The 5'-region lacks a typical TATA box, but has high homology to a transcription initiator consensus sequence. The proximal region is GC-rich, and a CpG island spans the putative transcription start site (Bernauer et al., 2001). Several upstream regulatory DNA motifs were identified, including AP2, NF-AT, C/EBP and Ahr/Arnt (Bernauer et al., 2001).

Although the rapid effects of progesterone are mediated at the cell surface membrane of a variety of types of cell in pig hepatocytes (Falkenstein et al., 1998) and in Chinese hamster ovary cells transfected with $\mathrm{mPR}$ (Falkenstein et al., 1999), progesterone binding is associated with the endo-membrane rather than the cell surface-membrane fraction. Nevertheless, the protein was exposed at the cell surface of human spermatozoa, as an antibody to the protein prevented induction of the acrosome reaction by progesterone, but not by other 
Table 2. Effects in vitro of progesterone on the ovary of domestic species

\begin{tabular}{|c|c|c|c|c|c|}
\hline \multirow[b]{2}{*}{ Species } & \multirow[b]{2}{*}{ Type of cell } & \multicolumn{2}{|c|}{ Effective dose of } & \multirow[b]{2}{*}{ Effect } & \multirow[b]{2}{*}{ Reference } \\
\hline & & Progestagen & Antiprogestagen & & \\
\hline \multirow[t]{3}{*}{ Pig } & Granulosa & $\begin{array}{l}1 \mathrm{pmol} \mathrm{P}^{-1} \\
1 \mathrm{pmol} \mathrm{P} \mathrm{I}^{-1}\end{array}$ & $\begin{array}{l}1 \mu \mathrm{mol} \text { RU486 } \mathrm{I}^{-1} \\
1 \mu \mathrm{mol} \text { RU486 } \mathrm{I}^{-1} \\
30 \mathrm{nmol} \text { RU486 } \mathrm{I}^{-1} \\
30 \mathrm{nmol} \text { ONA I-1 }\end{array}$ & $\begin{array}{l}\text { Increased }\left[\mathrm{Ca}^{2+}\right], \mathrm{IP}_{3} \\
\text { Increased }\left[\mathrm{Ca}^{2+}\right], \mathrm{IP}_{3}, \mathrm{PKC}-\beta 1 \\
\text { Upregulated gPR and inhibited } \mathrm{E}_{2} / \mathrm{P} \\
\quad \text { accumulation }\end{array}$ & 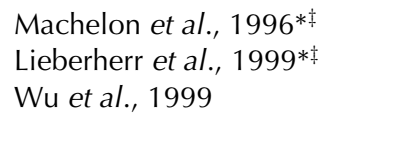 \\
\hline & Stable granulosa cell line (JC-410) & $\begin{array}{l}3-10 \mu \mathrm{mol} \mathrm{LNG} \mathrm{I}{ }^{-1} \\
10 \mu \mathrm{mol} \mathrm{P} \mathrm{I}-1 \\
0.1 \mu \mathrm{mol} \mathrm{RWJ} \mathrm{I}{ }^{-1}\end{array}$ & $1-10 \mu \mathrm{mol} R \cup 486 \mathrm{I}^{-1}$ & $\begin{array}{l}\text { LNG and RU stimulated P accumulation. } \\
\text { P, RU and LNG increased P450scc } \\
\text { transcription. RWJ inhibited P450scc } \\
\text { expression. }\end{array}$ & Swan et al., $2002^{\ddagger}$ \\
\hline & Theca & & $\begin{array}{l}30 \mathrm{nmol} \mathrm{RU} 486 \mathrm{I}^{-1} \\
30 \mathrm{nmol} \mathrm{ONA} \mathrm{I}{ }^{-1}\end{array}$ & Inhibited P secretion & Wu et al., 1999 \\
\hline \multirow[t]{2}{*}{ Sheep } & Ovarian surface epithelium cells & $3 \mathrm{nmol} \mathrm{P} \mathrm{I}{ }^{-1}$ & & $\begin{array}{l}\text { Upregulated p53 and inhibited } \\
\mathrm{E}_{2} \text {-stimulated proliferation }\end{array}$ & Murdoch and Van Kirk, $2002^{\dagger}$ \\
\hline & & $30 \mathrm{nmol} \mathrm{P} \mathrm{I}$ & $60 \mathrm{nmol} \mathrm{RU} 486 \mathrm{I}^{-1}$ & Increased PARP activity & $\begin{array}{l}\text { Murdoch, } 1998^{\dagger}, \\
\text { Murdoch et al., } 2001^{\dagger}\end{array}$ \\
\hline \multirow[t]{4}{*}{ Cow } & $\begin{array}{l}\text { Granulosa } \\
\text { (+ forskolin/insulin) }\end{array}$ & $1 \mu \mathrm{mol} \mathrm{MPA} \mathrm{I-1}$ & $\begin{array}{l}0.1 \mu \mathrm{mol} \mathrm{RU} 486 \mathrm{I}^{-1} \\
1 \mu \mathrm{mol} \mathrm{ONA} \mathrm{I}{ }^{-1}\end{array}$ & Inhibited upregulation of oxytocin gene & Lioutas et al., $1997^{\S}$ \\
\hline & Cumulus-oocyte complex & $10 \mu \mathrm{mol} \mathrm{P} \mathrm{^{-1 }}$ & & Inhibited $\mathrm{E}_{2}$ secretion & Mingoti et al., 2002 \\
\hline & Corpus luteum & $\left.50 \mathrm{nmol} \mathrm{P}\right|^{-1}$ & $\begin{array}{l}50 \mathrm{nmol} \mathrm{RU} 486 \mathrm{I}^{-1} \\
5 \mu \mathrm{mol} \mathrm{ONA} \mathrm{I}\end{array}$ & $\begin{array}{l}\text { P inhibited and RU and ONA stimulated } \\
\text { apoptosis induced by AG }\end{array}$ & Rueda et al., $2000^{\dagger}$ \\
\hline & & $5 \mu \mathrm{mol} \mathrm{P} \mathrm{I}$ & & Decreased $\mathrm{PGF}_{2 \alpha}$ and prostacyclin secretion & Pate, 1988 \\
\hline
\end{tabular}

*Demonstration of cell surface receptor action.

†Progestin effect reversed by gPR antagonist.

$\ddagger$ Progestin effect not reversed by gPR antagonist.

$\S$ Antiprogestin effect reversed by progestin.

AG: aminoglutethimide; $E_{2}$ : oestradiol; gPR: genomic progesterone receptor; IP: inositol trisphosphate; LNG: levonorgestrel; MPA: medroxyprogesterone acetate; ONA: onapristone (ZK98299); P: progesterone; P450scc: cytochrome P450 side-chain cleavage; PARP: pituitary adenyl cyclase activated regulatory protein; PKC- $\beta 1$ : protein kinase C $\beta 1$; RU486: mifepristone; RWJ: RWJ26819. 
Table 3. Effects in vitro of progesterone on the human ovary

\begin{tabular}{|c|c|c|c|c|}
\hline \multirow[b]{2}{*}{ Type of cell } & \multicolumn{2}{|c|}{ Effective dose range } & \multirow[b]{2}{*}{ Effect } & \multirow[b]{2}{*}{ Reference } \\
\hline & Progestagen & Antiprogestagen & & \\
\hline \multirow[t]{7}{*}{ Granulosa (luteinizing IVF cells) } & $1 \mathrm{nmol}-10 \mu \mathrm{mol} \mathrm{P} \mathrm{I}-1$ & $\begin{array}{l}50 \mu \mathrm{mol} \text { RU486 } \mathrm{I}^{-1} \\
10 \mu \mathrm{mol} \text { ORG }\end{array}$ & Induced apoptosis and stimulated caspase-3 & Svensson et al., $2001^{\S}$ \\
\hline & $10 \mathrm{nmol} \mathrm{P} \mathrm{I}{ }^{-1}$ & $1 \mu \mathrm{mol} \mathrm{RU} 486 \mathrm{I}^{-1}$ & $\begin{array}{l}\text { P reduced apoptosis and had protective effect } \\
\text { on survival }\end{array}$ & Makrigiannakis et al., $2000^{\dagger}$ \\
\hline & & $\begin{array}{l}50 \mu \mathrm{mol} \mathrm{RU} 486 \mathrm{I}^{-1} \\
50 \mu \mathrm{mol} \mathrm{HRP} \mathrm{I}{ }^{-1}\end{array}$ & Reduced secretion of $\left(\mathrm{E}_{2}\right) \mathrm{P}$ and relaxin & VanderVoort et al., 2000 \\
\hline & & $5-10 \mathrm{nmol}$ RU486 $\mathrm{I}^{-1}$ & Suppressed P secretion & DiMattina et al., 1986 \\
\hline & & $50-500 \mu \mathrm{mol} R \cup 486 \mathrm{I}^{-1}$ & Inhibited 17-hydroxylase. No effect on $E_{2}$ secretion & DiMattina et al., 1987 \\
\hline & & $10-100 \mathrm{nmol}$ RU486 I-1 & No effect on basal, hCG- or $\mathrm{PGE}_{2}$-stimulated P & Greenberg et al., 1990 \\
\hline & $640 \mathrm{nmol} \mathrm{P} \mathrm{I}$ & $64 \mathrm{nmol} \mathrm{RU486} \mathrm{\textrm {I } ^ { - 1 }}$ & $\mathrm{P}$ inhibited proliferation and differentiation & Chaffkin et al., $1993^{\dagger}$ \\
\hline Ovarian surface epithelium cells & $160 \mathrm{nmol} \mathrm{P} \mathrm{I^{-1 }}$ & $100 \mathrm{nmol}$ ORG $\mathrm{I}^{-1}$ & Suppressed proliferation. Enhanced proliferation & Ivarsson et al., $2001^{\dagger}$ \\
\hline Corpus luteum & & $10 \mathrm{nmol}$ RU486 I-1 & Inhibited hCG-stimulated (but not basal) P secretion & Ottander et al., 2000 \\
\hline SKOV-3 ovarian cancer & $30-300 \mathrm{nmol} \mathrm{P} \mathrm{I}$ & $3 \mu \mathrm{mol}$ RU486 $\mathrm{I}^{-1}$ & Inhibited uPA secretion and cell invasiveness & McDonnel and Murdoch, 2001 \\
\hline $\begin{array}{l}\text { gPR-positive ovarian carcinoma } \\
\text { cell lines }\end{array}$ & & $30-120 \mathrm{nmol}$ RU486 I $\mathrm{I}^{-1}$ & $\begin{array}{l}\text { Inhibited proliferation (blocked } G_{0} / G_{1} \text { phase } \\
\text { of cell cycle) and downregulated gPR }\end{array}$ & Rose and Barnea, 1996 \\
\hline
\end{tabular}

*Demonstration of cell surface receptor action.

†Progestin effect reversed by gPR antagonist.

$\ddagger$ Progestin effect not reversed by gPR antagonist.

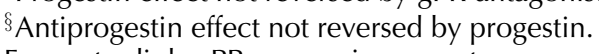

$\mathrm{E}_{2}$ : oestradiol; gPR: genomic progesterone receptor; HRP: horseradish peroxidase; ORG: ORG-5128; P: progesterone; PGE 2 : prostaglandin $\mathrm{E}_{2}$; RU486: mifepristone; SKOV-3: human ovarian cancer cell line; uPA: urokinase plasminogen activator. 
Table 4. Effects in vitro of progesterone on the rat ovary

\begin{tabular}{|c|c|c|c|c|}
\hline \multirow[b]{2}{*}{ Type of cell } & \multicolumn{2}{|c|}{ Effective dose range } & \multirow[b]{2}{*}{ Effect } & \multirow[b]{2}{*}{ Reference } \\
\hline & Progestagen & Antiprogestagen & & \\
\hline \multirow[t]{5}{*}{ Granulosa cells } & $\begin{array}{l}1 \mu \mathrm{mol} \mathrm{P} \mathrm{I}{ }^{-1} \\
1 \mu \mathrm{mol} \mathrm{R} 5020 \mathrm{I}^{-1}\end{array}$ & & $\begin{array}{l}\text { Enhanced LH- and FSH-stimulated } \\
\text { steroidogenesis }\end{array}$ & Fanjul et al., 1983 \\
\hline & & $\begin{array}{l}300 \mathrm{nmol} R \cup 486 \mathrm{I}^{-1} \\
30 \mu \mathrm{mol} R \cup 486 \mathrm{I}^{-1}\end{array}$ & $\begin{array}{l}\text { Inhibited aromatase. Inhibited } \\
\text { P secretion }\end{array}$ & Fortune and Vincent, 1983 \\
\hline & $200 \mu \mathrm{mol} \mathrm{P} \mathrm{I}-1$ & $64 \mu \mathrm{mol} \mathrm{RU} 486 \mathrm{I}^{-1}$ & $\begin{array}{l}\mathrm{P} \text { reduced and antagonist enhanced } \\
\text { apoptosis and cell adhesion }\end{array}$ & Peluso and Pappalardo, $1994^{\dagger}$ \\
\hline & & 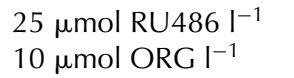 & $\begin{array}{l}\text { Antagonized hCG-induced apoptosis } \\
\text { and suppressed expression of gPR }\end{array}$ & Svensson et al., 2000 \\
\hline & & 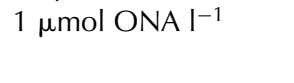 & $\begin{array}{l}\text { Inhibition of hCG- and forskolin-induced } \\
\text { PACAP mRNA expression }\end{array}$ & Ko et al., 1999 \\
\hline Large granulosa cells & $\begin{array}{l}640 \mathrm{nmol} \mathrm{P} \mathrm{I}-1 \\
64 \mathrm{nmol} \mathrm{P} \mathrm{I}^{-1}\end{array}$ & $64 \mu \mathrm{mol} R U 486 \mathrm{I}^{-1}$ & $\begin{array}{l}\text { P inhibited apoptosis of large GC } \\
\text { Stimulated bFGF secretion and inhibited } \\
\text { apoptosis in large luteal cells }\end{array}$ & $\begin{array}{l}\text { Luciano et al., } 1994^{\dagger} \\
\text { Peluso and Pappalardo, 1999* }\end{array}$ \\
\hline Small granulosa cells & $160 \mathrm{nmol} \mathrm{PI^{-1 }}$ & $64 \mu \mathrm{mol} \mathrm{RU} 486 \mathrm{I}^{-1}$ & $\mathrm{P}$ inhibited insulin-stimulated mitosis & Luciano and Peluso, $1995^{\dagger}$ \\
\hline Granulosa/SIGC & $640 \mathrm{nmol} \mathrm{PI^{-1 }}$ & $\begin{array}{l}640 \mathrm{nmol} \mathrm{RU} 486 \mathrm{I}^{-1} \\
640 \mathrm{nmol} \mathrm{ONA} \mathrm{I}{ }^{-1}\end{array}$ & Inhibited apoptosis and MAPKK & Peluso et al., 2001*‡ \\
\hline \multirow[t]{2}{*}{ Luteinizing granulosa cells } & & $10 \mu \mathrm{mol} \mathrm{ONA} \mathrm{I}^{-1}$ & $\begin{array}{l}\text { Inhibited hCG and forskolin on PAC-1 } \\
\text { receptor expression }\end{array}$ & Ko and Park-Sarge, 2000 \\
\hline & $150 \mathrm{nmol} \mathrm{PI^{-1 }}$ & $\begin{array}{l}2 \mu \mathrm{mol} \text { ONA I-1 } \\
2 \mu \mathrm{mol} \mathrm{RU} 486 \mathrm{I}^{-1}\end{array}$ & $\begin{array}{l}\text { Induced GRE expression; RU486 and } \\
\text { ONA antagonized P effect, but did not } \\
\text { block LH-induction of gPR }\end{array}$ & Natraj and Richards, $1993^{\dagger}$ \\
\hline CL cells & $10 \mu \mathrm{mol} \mathrm{LNG} \mathrm{I^{-1 }}$ & & $\begin{array}{l}\text { Inhibited LH and cAMP stimulated } \\
\text { P secretion }\end{array}$ & Telleria and Deis, 1994 \\
\hline $\mathrm{CL}$ of pregnancy & $\begin{array}{l}0.1 \mu \mathrm{mol} \mathrm{P} \mathrm{I}-1 \\
1-100 \mu \mathrm{mol} \mathrm{R} 5020 \mathrm{I}^{-1}\end{array}$ & & $\begin{array}{l}\text { Suppressed IL- } 6 \text { expression } \\
\text { Stimulated P secretion }\end{array}$ & $\begin{array}{l}\text { Telleria et al., } 1998 \\
\text { Telleria et al., } 1999\end{array}$ \\
\hline CL and SV40 sensitive cell line & $10 \mathrm{nmol} \mathrm{P} \mathrm{I-1}$ & $1 \mu \mathrm{mol}$ RU486 $\mathrm{I}^{-1}$ & Inhibited $20 \alpha-H S D$ expression & Sugino et al., $1997^{\dagger \S}$ \\
\hline CL-derived phagocytes & $300 \mathrm{nmol} \mathrm{P} \mathrm{I}$ & $100 \mathrm{nmol} R U 486 \mathrm{I}^{-1}$ & Inhibited superoxide production & Sugino et al., $1996^{\dagger}$ \\
\hline
\end{tabular}

*Demonstration of cell surface receptor action.

†Progestin effect reversed by gPR antagonist.

¥Progestin effect not reversed by gPR antagonist.

$\S$ Effect may be explained by action at the glucocorticoid receptor.

bFGF: basic fibroblast growth factor; CL: corpus luteum; GC: granulosa cell; gPR: genomic progesterone receptor; GRE: glucocorticoid response element; $20 \alpha-H S D$ : hydroxysteroid dehydrogenase; IL-6: interleukin 6; LNG: levonorgestrel; MAPKK: mitogen-activated protein kinase kinase; ONA: onapristone (ZK98299); ORG: ORG-5128; P: progesterone; PACAP: pituitary adenylate cyclase activating polypeptide; PAC-1: PACAP-specific receptor-1; RU486: mifepristone. 


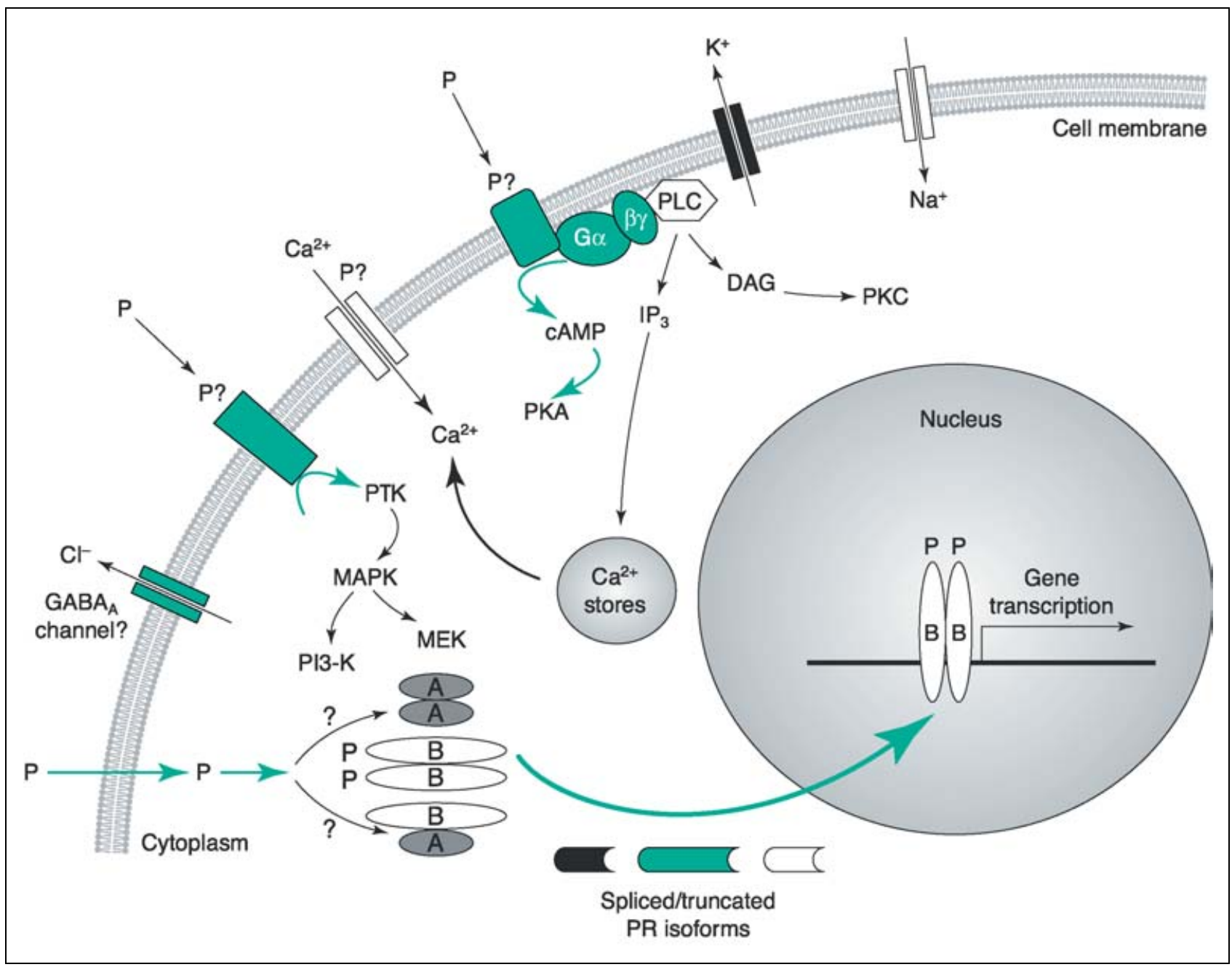

Fig. 2. Genomic and non-genomic activation pathways of progesterone. DAG: diacylglycerol; GABA: gamma aminobutyric acid; MAPK: mitogen-activated protein kinase; MEK: mitogen-activated (extracellular) protein kinase kinase; P: progesterone; PI3-K: phosphatidyl inositol 3-kinase; PKA: protein kinase A; PKC: protein kinase C; PLC: phospholipase C; PTK: protein tyrosine kinase.

agonists (Falkenstein et al., 1999; Buddhikot et al., 1999).

\section{What is the nature of the membrane receptor for progesterone?}

There is conflicting data on the nature of the membrane progesterone receptor, and the signalling pathways used in different types of cell (Fig. 2). Studies of rapid, membrane-mediated events in several types of cell have identified a wide range of changes, including $\mathrm{Ca}^{2+}$ mobilization (influx or mobilization from intracellular stores), opening of $\mathrm{Na}^{+}$and $\mathrm{Cl}^{-}$channels, and the activation of phospholipase $\mathrm{C}$ (leading to increased intracellular $\mathrm{IP}_{3}$ and diacylglycerol generation), Pertussis toxin-insensitive G-protein-coupled receptors, PKC, tyrosine kinase and MAPK pathways (for review, see Wehling, 1997; Falkenstein et al., 2000a). However, it is unclear which of these changes are primary, and which are activated by other downstream events or by cross-talk with other signalling pathways. Although the rat ovarian $\mathrm{mPR}$ has some similarity to gamma amino butyric acid $\mathrm{A}$ receptors $\left(G_{A B A}\right)$ (Peluso and Pappalardo, 1998), GABA agonists and antagonists failed to affect progesterone binding to bovine luteal membranes (Menzies et al., 1999).

The two most widely used models for studies of the non-genomic progesterone receptor are human spermatozoa (which had been thought to lack the genomic PR; Castilla et al., 1995) and the Xenopus oocyte (in which responses in enucleated cells, and membraneimpermeable derivatives of progesterone can mimic all the rapid changes induced by the steroid). However, doubts about these models have been raised as (a) spermatozoa appear to contain small amounts of gPR mRNA (Sachdeva et al., 2000; Contreras and Llanos, 
Table 5. Studies demonstrating expression of the gene for genomic progesterone receptor in ovarian tissues

\begin{tabular}{|c|c|c|c|c|}
\hline Species & Primers (nucleotides) & Method & Type of cell & Reference \\
\hline \multirow[t]{4}{*}{ Human } & $\begin{array}{l}2113-2343 \\
144-367\end{array}$ & $\begin{array}{l}\text { RT-PCR (PR-B) } \\
\text { RT-PCR (PR-A/B) } \\
\text { Southern blots/ISH }\end{array}$ & $\mathrm{CL}$ & Ottander et al., 2000 \\
\hline & $1341-1360$ & RT-PCR/Southern blot & $\mathrm{CL}$ & Misao et al., 1998a \\
\hline & PR-A and PR-B & Quantitative RT-PCR Southern blot & $\mathrm{CL}$ & Misao et al., $1998 \mathrm{~b}$ \\
\hline & $1817-36$ and $2330-49$ & RT-PCR & HOSE cells & Lau et al., 1999 \\
\hline \multirow[t]{3}{*}{ Monkey } & LBD & $\begin{array}{l}\text { RT-PCR, sequencing, restriction } \\
\text { enzyme cleavage }\end{array}$ & Germinal epithelium, CL, luteinizing GC & Chandrasekher et al., 1994 \\
\hline & LBD & RPAse & $\mathrm{CL}$, luteinized GC & Duffy et al., 1996 \\
\hline & LBD & Northern blot, RPAse & $\mathrm{CL}$ & Duffy and Stouffer, 1995 \\
\hline Baboon & Full length hPR & Northern blot & $\mathrm{CL}$ & Hild-Petito and Fazleabas, 1997 \\
\hline Bovine & Hinge/LBD & $\begin{array}{l}\text { RPAse/northern blot } \\
\text { RT-PCR, sequencing } \\
\text { Northern blot }\end{array}$ & $\begin{array}{l}\mathrm{GC} \\
\mathrm{CL} \\
\text { Periovulatory follicle }\end{array}$ & $\begin{array}{l}\text { Lioutas et al., } 1997 \\
\text { Rueda et al., } 2000 \\
\text { Cassar et al., } 2002\end{array}$ \\
\hline Rat & $\begin{array}{l}\text { LBD } \\
\text { LBD }\end{array}$ & $\begin{array}{l}\text { RT-PCR, ISH } \\
\text { RT-PCR }\end{array}$ & $\begin{array}{l}\text { GC of preovulatory follicles } \\
\text { Transient expression in GC of luteinizing } \\
\text { primed ovary }\end{array}$ & $\begin{array}{l}\text { Park-Sarge et al., } 1995 \\
\text { Park and Mayo, } 1991\end{array}$ \\
\hline
\end{tabular}

CL: corpus luteum; GC: granulosa cell; HOSE: human ovarian surface epithelium cells; hPR: human progesterone receptor; ISH: in situ hybridization; LBD: ligand-binding domain; PR-A/B; progesterone receptor-A or -B; RPAse: ribonuclease protection assay. 
Table 6. Studies demonstrating genomic progesterone receptor protein expression in ovarian tissues

\begin{tabular}{|c|c|c|c|c|c|}
\hline Species & Antibody & Protein detected & Type of cell & Subcellular localization & Reference \\
\hline \multirow[t]{11}{*}{ Human } & Range of mAbs & 116 and $97 \mathrm{kDa}$ & $\begin{array}{l}\text { Ovarian tumours, } \mathrm{CL} \text {, stroma, HOSE } \\
\text { GC of dominant follicles }\end{array}$ & Nuclear & $\begin{array}{l}\text { Press and Green, } 1988 \\
\text { Iwai et al., } 1990\end{array}$ \\
\hline & $\mathrm{mAb} C 262$ & 120 and $90 \mathrm{kDa}$ & $\mathrm{CL}$ & Nuclear? & Misao et al., 1998a \\
\hline & $\mathrm{mAb} C 262$ & 116 and $90 \mathrm{kDa}$ & $\mathrm{CL}$ & & Misao et al., 1998b \\
\hline & Neomarkers hPRa3 & PR-B and PR-A & Ovarian cancers & Nuclear & Akahira et al., 2000 \\
\hline & Neomarkers hPRa7 & PR-A & & & \\
\hline & Neomarkers hPRa2 & PR-B & & & \\
\hline & Novocastra mAb & 120 and $90 \mathrm{kDa}$ & Theca and small and large luteal cells & Nuclear & Suzuki et al., 1994 \\
\hline & JZB39 mAb & & $60-80 \%$ of luteinizing GC & Nuclear & Greenberg et al., 1990 \\
\hline & Abbott K68 & & Germinal epithelium, $\mathrm{CL}$, cancer & Nuclear & Zeimet et al., 1994 \\
\hline & Abbott & & CL (theca; GC of some follicles) & & Revelli et al., 1996 \\
\hline & Abbott & & $\mathrm{CL}$ & Nuclear & Ottander et al., 2000 \\
\hline \multirow[t]{2}{*}{ Monkey } & JZB39 mAb & & $\begin{array}{l}\text { Germinal epithelium, stroma, theca, } \mathrm{CL} \text {, } \\
\text { GC of preovulatory follicles }\end{array}$ & Nuclear & Hild-Petito et al., 1988 \\
\hline & JZB39 mAb & & Luteinized GC & Nuclear & Duffy et al., 1996 \\
\hline Baboon & JZB39 mAb & & $\mathrm{CL}$ & Nuclear & Hild-Petito and Fazleabas, 1997 \\
\hline \multirow[t]{6}{*}{ Rat } & mAb C262 & $60 \mathrm{kDa}$ & GC/SIGC & Cell membrane & Peluso and Pappalardo, 1998 \\
\hline & $\mathrm{mAb} C 262$ & & Small GC & Cell membrane & Peluso et al., 2001 \\
\hline & mAb 928 & 115 and $85 \mathrm{kDa}$ & Luteinizing GC in culture & Nuclear & Natraj and Richards, 1993 \\
\hline & $\mathrm{mAb} 928$ & 97 and $66 \mathrm{kDa}$ & & & \\
\hline & $\mathrm{mAb} \mathrm{H} 928$ & & Not present in CL of pregnancy & & Park-Sarge et al., 1995 \\
\hline & & & $\begin{array}{l}\text { Transient expression in GC } \\
\text { of luteinizing ovary }\end{array}$ & & Park and Mayo, 1991 \\
\hline Pregnant rat & mAb LET81 & & $\mathrm{GC}$ and theca but not $\mathrm{CL}$ & Nuclear? & Telleria et al., 1999 \\
\hline Pig & Novocastra mAb & 120 and $80 \mathrm{kDa}$ & $\mathrm{CL}$, theca, $\mathrm{GC}$ of preovulatory follicles & Nuclear & Slomczynska et al., 2000 \\
\hline \multirow[t]{2}{*}{ Bovine } & JZB39 mAb & & Large and small luteal cells & Nuclear & Rueda et al., 2000 \\
\hline & $\mathrm{mAb} C 262$ & 60 and $55 \mathrm{kDa}$ & $\mathrm{CL}$ & Cell membrane & Bramley et al., 2002 \\
\hline
\end{tabular}

CL: corpus luteum; GC: granulosa cells; HOSE: human ovarian surface epithelium cells; mAb: monoclonal antibody; PR-A: progesterone receptor A; PR-B: progesterone receptor B; SIGC: spontaneously induced granulosa cells.

Epitopes: mAb C262: raised to C-terminal 14 amino acids of gPR; mAb 928: raised to hinge region of gPR; conformation-dependent; mAb JZB39: recognizes occupied and unoccupied human gPR. 
2001), and (b) cloning of the receptor responsible for inducing oocyte maturation (xPR) has revealed it to be similar to the mammalian genomic progesterone receptor (Maller, 2001). xPR associates with p42 MAPK and activates oocyte maturation non-classically by signalling through the PI3-K pathway (Bagowski et al., 2001).

Studies designed to identify and localize progesterone receptors in ovarian tissues have often used antibodies to the LBD of the gPR (Tables 5 and 6). However, antibodies raised to other regions of the gPR often give little or no signal in these same tissues (for review, see Press and Greene, 1988; El-Hefnawy et al., 2000; Luconi et al., 1998 - but see Luconi et al., 2002). Furthermore, a commonly used monoclonal antibody raised to the C-terminal end of the gPR (mAb C262) also detects immunoactivity in isolated rat granulosa cell membranes (Peluso and Pappalardo, 1998, 1999; Peluso et al., 2001) and in bovine luteal membranes and detergent extracts (Bramley et al., 2002), indicating that the membrane steroid receptor may possess a domain with homology to the LBD of the gPR. Indeed, different methods of detecting $\mathrm{mPR}$ can identify proteins of markedly different sizes even in the same tissues and species (for review, see Bramley et al., 2002). Finally, it will be of interest to establish whether gPR variants with deleted exons or novel 5'-regions (vide infra) are present in tissues with high amounts of membrane progesterone binding sites and, if so, to study their subcellular distribution at different stages of ovarian development and functional activity.

\section{Conclusion}

Progesterone can induce rapid and specific changes in a variety of tissues by acting via membrane-associated receptors. However, the nature of these receptors is presently unclear. Although membrane-associated, progesterone-specific receptors have been isolated and cloned from a range of tissues in a number of species, the protein isolated appears to be only one subunit of the (possibly dimeric) membrane receptor, and it has been difficult to demonstrate binding to the expressed protein. However, other studies indicate a membrane protein (or an associated protein) with homology to the ligand-binding domain of the genomic progesterone receptor. Further studies of these receptors in the ovary, and the ways in which they interact with other autocrine or paracrine factors, may lead to exciting advances in the understanding of the progesterone-dependent processes of ovulation, luteinization, follicular atresia and maternal recognition of pregnancy, and so permit the development of new methods of manipulating ovarian activity and fertility in humans and other mammalian species.

The author is most grateful to M. Rae and G. Scobie for their helpful discussions and comments, and to G. Menzies, for his excellent technical support.

\section{References}

Key references are identified by asterisks.

Akahira J, Inoue T, Suzuki T et al. (2000) Progesterone receptor isoforms $A$ and $B$ in human epithelial ovarian carcinoma: immunohistochemical and RT-PCR studies British Journal of Cancer 83 1488-1494

Bagowski C, Myers JW and Ferrell JE, Jr (2001) The classical progesterone receptor associates with p42 MAPK and is involved in phosphatidylinositol 3-kinase signaling in Xenopus oocytes Journal of Biological Chemistry 27637 708-37 714

Bernauer S, Wehling M, Gerdes D and Falkenstein E (2001) The human membrane progesterone receptor gene: genomic structure and promoter analysis DNA Sequence 12 13-25

Boonyaratanakornkit V, Scott MP, Ribon V, Sherman L, Anderson SM, Maller JL, Miller WT and Edwards DP (2001) Progesterone receptor contains a proline-rich motif that directly interacts with $\mathrm{SH} 3$ domains and activates C-Src family tyrosine kinases Molecular Cell 8 269-280

Bramley TA, Menzies GS, Rae MT and Scobie G (2002) Non-genomic steroid receptors in the bovine ovary Domestic Animal Endocrinology 23 1-10

Buddhikot M, Falkenstein E, Wehling M and Meizel S (1999) Recognition of a human sperm surface protein involved in the progesterone-initiated acrosome reaction by antisera against an endomembrane progesterone binding protein from porcine liver Molecular and Cellular Endocrinology 158 187-193

Cassar CA, Dow MPD, Pursley JR and Smith GW (2002) Effect of the preovulatory $\mathrm{LH}$ surge on bovine follicular progesterone receptor mRNA expression Domestic Animal Endocrinology 22 179-187

Castilla JA, Gil T, Molina J, Hortas ML, Rodriguez F, Torres-Munoz J, Vergara F and Herruzo AJ (1995) Undetectable expression of genomic progesterone receptor in human spermatozoa Human Reproduction 10 $1757-1760$

Cenedella RJ, Sexton PS and Zhu XL (1999) Lens epithelia contain a high-affinity, membrane steroid hormone-binding protein Investigative Ophthalmology and Visual Science 40 1452-1459

Chaffin CL and Stouffer RL (2000) Role of gonadotrophins and progesterone in the regulation of morphological remodelling and atresia in the monkey peri-ovulatory follicle Human Reproduction 15 2489-2495

Chaffkin LM, Luciano AA and Peluso JJ (1993) The role of progesterone in regulating human granulosa cell proliferation and differentiation in vitro. Journal of Clinical Endocrinology and Metabolism 76 696-700

Chandrasekher YA, Melner MH, Nagalla SR and Stouffer RL (1994) Progesterone receptor, but not estradiol receptor, messenger ribonucleic acid is expressed in luteinizing granulosa cells and the corpus luteum in rhesus monkeys Endocrinology 135 307-314

Cohen-Solal K, Bailly A, Rauch C, Quesne M and Milgrom E (1993) Specific binding of progesterone receptor to progesterone-responsive elements does not require prior dimerization European Journal of Biochemistry 214 189-195

Contreras HR and Llanos MN (2001) Detection of progesterone receptors in human spermatazoa and their correlation with morphological and functional properties International Journal of Andrology 24 246-252

Di Mattina M, Albertson B, Seyler DE, Loriaux DL and Falk R (1986) Effect of the antiprogestin RU486 on progesterone production by cultured human granulosa cells: inhibition of the ovarian $3 \beta$-hydroxysteroid dehydrogenase Contraception 34 199-206

Di Mattina M, Albertson BD, Tyson V, Loriaux DL and Falk RJ (1987) Effect of the antiprogestin RU486 on human ovarian steroidogenesis Fertility and Sterility 48 229-233

Duffy DM and Stouffer RL (1995) Progesterone receptor messenger ribonucleic acid in the primate corpus luteum during the menstrual cycle: possible regulation by progesterone Endocrinology 1361869 1876

Duffy DM, Molskness TA and Stouffer RL (1996) Progesterone receptor messenger ribonucleic acid and protein in luteinized granulosa cells of Rhesus monkeys are regulated in vitro by gonadotropins and steroids Biology of Reproduction $\mathbf{5 4}$ 888-895

El-Hefnawy T, Manna PR, Luconi M, Baldi E, Slotte JP and Huhtaniemi I (2000) Progesterone action in a murine Leydig tumor cell line 
(mLTC-1), possibly through a nonclassical receptor type Endocrinology $141247-255$

Falkenstein E, Meyer C, Eisen C, Scriba PC and Wehling M (1996) Fulllength cDNA sequence of a progesterone membrane-binding protein from porcine vascular smooth muscle cells Biochemical and Biophysical Research Communications 229 86-89

Falkenstein E, Schmieding K, Lange A, Meyer C, Gerdes D, Welsch U and Wehling M (1998) Localization of a putative progesterone membrane binding protein in porcine hepatocytes Cellular and Molecular Biology 44 571-578

Falkenstein E, Heck M, Gerdes D, Grube D, Christ M, Weigel M, Buddhikot M, Meizel S and Wehling M (1999) Specific progesterone binding to a membrane protein and related nongenomic effects on $\mathrm{Ca}^{2+}{ }^{2}$ fluxes in sperm Endocrinology 140 5999-6002

Falkenstein E, Tillmann H-C, Christ M, Feuring M and Wehling M (2000a) Multiple actions of steroid hormones - a focus on rapid, nongenomic effects Pharmacological Reviews 52 513-556

*Falkenstein E, Norman AW and Wehling M (2000b) Mannheim classification of nongenomically initiated (rapid) steroid action(s) Journal of Clinical Endocrinology and Metabolism 85 2072-2075

Fanjul LF, Ruiz de Galarreta CM and Hsueh AJ (1983) Progestin augmentation of gonadotropin-stimulated progesterone production by cultured rat granulosa cells Endocrinology 112 405-407

Fortune JE and Vincent SE (1983) Progesterone inhibits the induction of aromatase activity in rat granulosa cells in vitro. Biology of Reproduction 28 1078-1089

Gerdes D, Wehling M, Leube B and Falkenstein E (1998) Cloning and tissue expression of two putative steroid membrane receptors Biological Chemistry 379 907-911

Giangrande PH and McDonnell DP (1999) The A and B isoforms of the human progesterone receptor: two functionally different transcription factors encoded by a single gene Recent Progress in Hormone Research 54 291-314

Greenberg LH, Stouffer RL, Brenner RM, Molskness TA, Hild-Petito SA and Yu Q (1990) Are human luteinizing granulosa cells a site of action for progesterone and relaxin? Fertility and Sterility $\mathbf{5 3}$ 446-453

Hild-Petito S and Fazleabas AT (1997) Expression of steroid receptors and steroidogenic enzymes in the baboon (Papio anubis) corpus luteum during the menstrual cycle and early pregnancy Journal of Clinical Endocrinology and Metabolism 82 955-962

Hild-Petito S, Stouffer RL and Brenner RM (1988) Immunocytochemical localization of estradiol and progesterone receptors in the monkey ovary throughout the menstrual cycle Endocrinology 123 2896-2905

Hirata S, Shoda T, Kato J and Hoshi K (2000) The novel form of the progesterone receptor CDNA in the human testis and detection of its mRNA in the human uterine endometrium Oncology 59 Supplement 1 $39-44$

Hirata S, Shoda T, Kato J and Hoshi K (2002) The novel exon, exon T, of the human progesterone receptor gene and the genomic organization of the gene Journal of Steroid Biochemistry and Molecular Biology $\mathbf{8 0}$ 299-305

Hodges YK, Richer JK, Horwitz HB and Horwitz LD (1999) Variant estrogen and progesterone receptor messages in human vascular smooth muscle Circulation 99 2688-2693

Ivarsson K, Sundfeldt K, Brannstrom M and Janson PO (2001) Production of steroids by human ovarian surface epithelial cells in culture: possible role of progesterone as growth inhibitor Gynecologic Oncology 82 116121

Iwai T, Nanbu Y, Iwai M, Taii S, Fijii S and Mori T (1990) Immunocytochemical localization of oestrogen receptors and progesterone receptors in the human ovary throughout the menstrual cycle Virchows Archives A Pathology, Anatomy and Histopathology 417 369-375

Kelly MJ and Levine ER (2001) Rapid actions of plasma membrane estrogen receptors Trends in Endocrinology and Metabolism 12 152-156

Ko C and Park-Sarge O-K (2000) Progesterone receptor activation mediates LH-induced type-1 pituitary adenylate cyclase activating polypeptide receptor (PAC1) gene expression in rat granulosa cells Biochemical and Biophysical Research Communications 277 270-279
Ko C, In Y-H and Park-Sarge O-K (1999) Role of progesterone receptor activation in pituitary adenylate cyclase activating polypeptide gene expression in rat ovary Endocrinology 140 5185-5194

Krebs CJ, Jarvis ED, Chan J, Lydon JP, Ogawa S and Pfaff DW (2000) A membrane-associated progesterone-binding protein, 25-Dx, is regulated by progesterone in brain regions involved in female reproductive behaviors Proceedings National Academy of Sciences USA 9712816 12821

Lau K-M, Mok SC and Ho S-M (1999) Expression of human estrogen receptor-a and $-b$, progesterone receptor, and androgen receptor mRNA in normal and malignant ovarian epithelial cells Proceedings National Academy of Sciences USA 96 5722-5727

Le Mellay V, Lasmoles $\mathbf{F}$ and Lieberherr $\mathbf{M}(1999) \mathrm{G} \alpha_{q / 11}$ and $\mathrm{G} \beta \gamma$ proteins and membrane signaling of calcitriol and estradiol Journal of Cellular Biochemistry 75 138-146

* Lieberherr M, Grosse B and Machelon V (1999) Phospholipase C- $\beta$ and ovarian sex steroids in pig granulosa cells Journal of Cellular Biochemistry 74 50-60

Lioutas C, Einspanier A, Kascheike B, Walther N and Ivell R (1997) An autocrine progesterone positive feedback loop mediates oxytocin upregulation in bovine granulosa cells during luteinization Endocrinology 138 5059-5062

Luciano AM and Peluso JJ (1995) Effect of in vivo gonadotropin treatment on the ability of progesterone, estrogen, and cyclic adenosine 5'-monophosphate to inhibit insulin-dependent granulosa cell mitosis in vitro. Biology of Reproduction 53 664-669

Luciano AM, Pappalardo A, Ray C and Peluso JJ (1994) Epidermal growth factor inhibits large granulosa cell apoptosis by stimulating progesterone synthesis and regulating the distribution of intracellular free calcium Biology of Reproduction 51 646-654

Luconi M, Bonaccorsi L, Maggi M, Pecchioli P, Krausz C, Forti G and Baldi E (1998) Identification and characterization of functional nongenomic progesterone receptors on human sperm membrane Journal of Clinical Endocrinology and Metabolism 83 877-885

Luconi M, Bonaccorsi L, Bini L, Liberatori S, Pallini V, Forti G and Baldi E (2002) Characterization of membrane nongenomic receptors for progesterone in human spermatazoa Steroids 67 505-509

McDonnel AC and Murdoch WJ (2001) High-dose progesterone inhibition of urokinase secretion and invasive activity by SKOV-3 ovarian carcinoma cells: evidence for a receptor-independent nongenomic effect on the plasma membrane Journal of Steroid Biochemistry and Molecular Biology 78 185-191

Machelon V, Nome F, Grosse B and Lieberherr M (1996) Progesterone triggers rapid transmembrane calcium influx and/or calcium mobilization from endoplasmic reticulum, via a Pertussis-insensitive G-protein in granulosa cells in relation to luteinization process Journal of Cell Biochemistry $61619-628$

Makrigiannakis A, Coukos G, Christofidou-Solomidou M, Montas S and Coutifaris C (2000) Progesterone is an autocrine/paracrine regulator of human granulosa cell survival in vitro. Annals of the New York Academy of Science $\mathbf{9 0 0} 16-25$

Maller JL (2001) The elusive progesterone receptor in Xenopus oocytes Proceedings National Academy of Sciences USA 98 8-10

Menzies GS, Howland K, Rae MT and Bramley TA (1999) Stimulation of binding of $\left[{ }^{3} \mathrm{H}\right]$-progesterone to bovine luteal cell-surface membranes: specificity of digitonin Molecular and Cellular Endocrinology 153 57-69

Meyer C, Schmid R, Scriba PC and Wehling M (1996) Purification and partial sequencing of high-affinity progesterone binding site(s) from porcine liver membranes European Journal of Biochemistry 239 726-731

Mingoti GZ, Garcia JM and Rosa-e-Silva AAM (2002) Steroidogenesis in cumulus cells of bovine cumulus-oocyte complexes matured in vitro with BSA and different concentrations of steroids Animal Reproduction Science 69 175-186

Misao R, Sun WS, Iwagaki S, Fujimoto J and Tamaya T (1998a) Identification of various exon-deleted progesterone receptor mRNAs in human endometrium and ovarian endometriosis Biochemical and Biophysical Research Communications 252 302-306 
Misao R, Nakanishi Y, Fujimoto J and Tamaya T (1998b) Steroid receptor mRNA levels in human corpus luteum Hormone Research 50 155-159

Misao R, Nakanishi Y, Iwagaki S, Fujimoto J and Tamaya T (1998c) Expression of progesterone receptor isoforms in corpora lutea of human subjects: correlation with serum estrogen and progesterone concentrations Molecular Human Reproduction 4 1045-1052

Misao R, Nakanishi Y, Sun W-S, Iwagaki S, Fujimoto J and Tamaya T (2000) Identification of exon-deleted progesterone receptor mRNAs in human uterine endometrial cancers Oncology 58 60-65

Mote PA, Balleine RL, McGowan EM and Clarke CL (1999) Colocalization of progesterone receptors $\mathrm{A}$ and $\mathrm{B}$ by dual immunofluorescent histochemistry in human endometrium during the menstrual cycle Journal of Clinical Endocrinology and Metabolism 84 2963-2971

Murdoch WJ (1998) Perturbation of sheep ovarian surface epithelial cells by ovulation: evidence for roles of progesterone and poly(ADP-ribose) polymerase in the restoration of DNA integrity Journal of Endocrinology $156503-508$

Murdoch WJ and Van Kirk EA (2002) Steroid hormonal regulation of proliferative, p53 tumor suppressor, and apoptotic responses of sheep ovarian surface epithelial cells Molecular and Cellular Endocrinology 186 61-67

Murdoch WJ, Townsend RS and McDonnel AC (2001) Ovulation-induced DNA damage in ovarian surface epithelial cells of ewes: prospective regulatory mechanisms of repair/survival and apoptosis Biology of Reproduction 65 1417-1424

Natraj U and Richards JS (1993) Hormonal regulation, localization, and functional activity of the progesterone receptor in granulosa cells of rat preovulatory follicles Endocrinology 133 761-769

Nolte I, Jeckel D, Wieland FT and Sohn K (2000) Localization and topology of ratp28, a member of a novel family of putative steroid-binding proteins Biochimica et Biophysica Acta 1543 123-130

Orti E, Bodwell JE and Munck A (1992) Phosphorylation of steroid hormone receptors Endocrine Reviews 13 105-128

Ottander U, Hosokawa K, Liu K, Bergh A, Ny T and Olofsson JI (2000) A putative stimulatory role of progesterone acting via progesterone receptors in the steroidogenic cells of the human corpus luteum Biology of Reproduction 62 655-663

Park O-K and Mayo KE (1991) Transient expression of progesterone receptor messenger RNA in ovarian granulosa cells after the preovulatory luteinizing hormone surge Molecular Endocrinology 5 967-978

Park-Sarge O-K, Parmer TG, Gu Y and Gibori G (1995) Does the rat corpus luteum express the progesterone receptor? Endocrinology 1361537 1543

Pate JL (1988) Regulation of prostaglandin synthesis by progesterone in the bovine corpus luteum Prostaglandins 36 303-315

Peluso JJ and Pappalardo A (1994) Progesterone and cell-cell adhesion interact to regulate rat granulosa cell apoptosis Biochemical Cell Biology 72 547-551

Peluso JJ and Pappalardo A (1998) Progesterone mediates its anti-mitogenic and anti-apoptotic actions in rat granulosa cells through a progesteronebinding protein with gamma aminobutyric acid A receptor-like features Biology of Reproduction 58 1131-1137

Peluso JJ and Pappalardo A (1999) Progesterone maintains large rat granulosa cell viability indirectly by stimulating small granulosa cells to synthesize basic fibroblast growth factor Biology of Reproduction $\mathbf{6 0}$ 290-296

*Peluso JJ, Fernandez G, Pappalardo A and White BA (2001) Characterization of a putative membrane receptor for progesterone in rat granulosa cells Biology of Reproduction 65 94-101

Pietras RJ and Szego CM (1977) Specific binding sites for estrogen at the outer surfaces of isolated endometrial cells Nature (London) 265 69-72

Press MF and Greene GL (1988) Localization of progesterone receptor with monoclonal antibodies to the human progestin receptor Endocrinology 122 1165-1175

Rae MT, Menzies GS, McNeilly AS, Woad K, Webb R and Bramley TA (1998) Specific non-genomic, membrane-localized binding sites for progesterone in the bovine corpus luteum Biology of Reproduction $\mathbf{5 8}$ 1394-1406
Revelli A, Pacchioni D, Cassoni P, Bussolati G and Massobrio M (1996) In situ hybridization study of messenger RNA for estrogen receptor and immunohistochemical detection of estrogen and progesterone receptors in the human ovary Gynecological Endocrinology 10 177-186

Revelli A, Massobrio M and Tesarik J (1998) Nongenomic actions of steroid hormones in reproductive tissues Endocrine Reviews 19 3-17

* Richer JK, Lange CA, Manning NG, Owen G, Powell R and Horwitz KB (1998) Convergence of progesterone with growth factor and cytokine signaling in breast cancer: progesterone receptors regulate signal transducers and activators of transcription expression and activity Journal of Biological Chemistry $27331317-31326$

Richer JK, Jacobsen BM, Manning NG, Abel MG, Wolf DM and Horwitz KB (2002) Differential gene regulation by the two progesterone receptor isoforms in human breast cancer cells Journal of Biological Chemistry 277 5209-5218

Rose FV and Barnea ER (1996) Response of human ovarian carcinoma cell lines to antiprogestin mifepristone Oncogene 12 999-1003

Rowan BG and O'Malley BW (2000) Progesterone receptor coactivators Steroids 65 545-549

Rueda BR, Hendry IR, Hendry WJ III, Stormshak F, Slayden OD and Davis JS (2000) Decreased progesterone levels and progesterone receptor antagonists promote apoptotic cell death in bovine luteal cells Biology of Reproduction 62 269-276

Sachdeva G, Shah CA, Kholkute SD and Puri CP (2000) Detection of progesterone receptor transcript in human spermatazoa Biology of Reproduction 62 1610-1614

Schmidt BMW, Gerdes D, Feuring M, Falkenstein E, Christ $M$ and Wehling M (2000) Rapid, nongenomic steroid actions: a new age? Frontiers in Neuroendocrinology 21 57-94

Selmin O, Lucier GW, Clark GC, Tritscher AM, Vanden Heuvel JP, Gastel JA, Walker NJ, Sutter TR and Bell DA (1996) Isolation and characterisation of a novel gene induced by $2,3,7,8$-tetrachlorodibenzo-p-dioxin in rat liver Carcinogenesis 17 2609-2615

Slomczynska M, Krok M and Pierscinski A (2000) Localization of the progesterone receptor in the porcine ovary Acta Histochemica 102 183-191

Smith CL, Wolford RG, O'Neill TB and Hager GL (2000) Characterization of transiently and constitutively expressed progesterone receptors: evidence for two functional states Molecular Endocrinology 14 956971

Smith DF (2000) Chaperones in progesterone receptor complexes Seminars in Cell and Developmental Biology 11 45-52

Sugino N, Shimamura K, Tamura H, Ono M, Nakamura Y, Ogino K and Kato $\mathbf{H}$ (1996) Progesterone inhibits superoxide radical production by mononuclear phagocytes in pseudopregnant rats Endocrinology 137 $749-754$

Sugino N, Telleria CM and Gibori G (1997) Progesterone inhibits $20 \alpha-$ hydroxysteroid dehydrogenase expression in the rat corpus luteum through the glucocorticoid receptor Endocrinology 138 4497-4500

Sutter-Dub M-T (2002) Rapid non-genomic and genomic responses to progestogens, estrogens, and glucocorticoids in the endocrine pancreatic B cell, the adipocyte and other cell types Steroids 67 77-93

Suzuki T, Sasano H, Kimura N, Tamura M, Fukaya T, Yajima A and Nagura H (1994) Immunohistochemical distribution of progesterone, androgen and oestrogen receptors in the human ovary during the menstrual cycle: relationship to expression of steroidogenic enzymes Human Reproduction 9 1589-1595

Svensson ECh, Markstrom E, Andersson M and Billig H (2000) Progesterone receptor-mediated inhibition of apoptosis in granulosa cells isolated from rats treated with human chorionic gonadotropin Biology of Reproduction 63 1457-1464

Svensson ECh, Markstrom E, Shao R, Andersson M and Billig H (2001) Progesterone receptor antagonists Org 31710 and RU 486 increase apoptosis in human periovulatory granulosa cells Fertility and Sterility 76 1225-1231

Swan CL, Agostini MC, Bartlewski PM, Feyles V, Urban RJ and Chedrese PJ (2002) Effects of progestins on progesterone synthesis in a stable 
porcine granulosa cell line: control of transcriptional activity of the cytochrome P450 side-chain cleavage gene Biology of Reproduction $\mathbf{6 6}$ 959-965

Telleria CM and Deis RP (1994) Effect of RU486 on ovarian progesterone production at pro-oestrus and during pregnancy: a possible dual regulation of the biosynthesis of progesterone Journal of Reproduction and Fertility 102 379-384

Telleria CM, Ou J, Sugino N, Ferguson S and Gibori G (1998) The expression of interleukin-6 in the pregnant rat corpus luteum and its regulation by progesterone and glucocorticoid Endocrinology 139 3597-3605

Telleria CM, Stocco CO, Stati AO and Deis RP (1999) Progesterone receptor is not required for progesterone action in the rat corpus luteum of pregnancy Steroids $64760-766$

VandeVoort CA, Overstreet JW, Lasley BL and Stewart DR (2000) Effects of progesterone receptor blockers on human granulosa-luteal cell culture secretion of progesterone, estradiol, and relaxin Biology of Reproduction 62 200-205

Vegeto E, Shabbaz M, Wen DX, Goldman M, O'Malley BW and McDonnell DP (1993) Human progesterone receptor $A$ form is a cell- and promoterspecific repressor of human progesterone receptor B function Molecular Endocrinology 7 1244-1255
Wehling M (1997) Specific, nongenomic actions of steroid hormones Annual Reviews of Physiology 59 365-393

Wei LL and Miner R (1994) Evidence for the existence of a third progesterone receptor protein in human breast cancer cell line T47D Cancer Research 54 340-343

Wei LL, Norris BM and Baker CJ (1997) An N-terminally truncated third progesterone receptor protein, $\mathrm{PR}(\mathrm{C})$, forms heterodimers with $\mathrm{PR}(\mathrm{B})$ but interferes in $\mathrm{PR}(\mathrm{B})-\mathrm{DNA}$ binding Journal of Steroid Biochemistry and Molecular Biology 62 287-297

Weigel NL and Zhang Y (1998) Ligand-independent activation of steroid hormone receptors Journal of Molecular Medicine 76 469-479

Wu ER, Wang JZ, Zhao JL and Xiao BL (1999) Flow cytometry of porcine ovarian cells: antiprogestins play an important role in progesterone receptor upregulation Gynecological Endocrinology 13 333-339

Zeimet AG, Muller-Holzner E, Marth C and Daxenbichler G (1994) Immunocytochemical versus biochemical receptor determination in normal and tumorous tissues of the female reproductive tract and breast Journal of Steroid Biochemistry and Molecular Biology 49 365-372

*Zhu XL, Sexton PS and Cenedella RJ (2001) Characterization of membrane steroid binding protein mRNA and protein in lens epithelial cells Experimental Eye Research 73 213-219 